\title{
Détruire les temples pour construire les écoles : reconstitution d'un objet historique
}

Destroy Temples to Build Schools: Constructing a Historical Object

廟座辦學: 一個史學對象的構建

\section{Vincent Goossaert}

\section{OpenEdition}

\section{Journals}

Édition électronique

URL : http://journals.openedition.org/extremeorient/180

DOI : $10.4000 /$ extremeorient. 180

ISSN : 2108-7105

\section{Éditeur}

Presses universitaires de Vincennes

Édition imprimée

Date de publication : 1 novembre 2011

Pagination : $35-51$

ISBN : 978-2-84292-334-1

ISSN : 0754-5010

\section{Référence électronique}

Vincent Goossaert, « Détruire les temples pour construire les écoles : reconstitution d'un objet

historique », Extrême-Orient Extrême-Occident [En ligne], 33 | 2011, mis en ligne le 01 novembre 2014 consulté le 19 avril 2019. URL : http://journals.openedition.org/extremeorient/180 ; DOI : 10.4000/ extremeorient. 180 


\title{
Détruire les temples pour construire les écoles : reconstitution d'un objet historique
}

\author{
Vincent Goossaert
}

«Construire les écoles avec les biens des temples», miaochan banxue, est un objet historique curieux ${ }^{1}$. Le slogan désigne un ensemble d'idées et de pratiques liées tant à l'introduction d'un système éducatif moderne en Chine au début du $\mathrm{Xx}^{\mathrm{e}}$ siècle, lequel nécessita d'édifier et de faire fonctionner des centaines de milliers d'écoles dans un pays appauvri et sans pouvoir central fort, qu'à des campagnes de réforme religieuse d'inspirations très diverses, allant d'un réformisme confucianiste à l'athéisme communiste en passant par l'activisme missionnaire chrétien, le tout vaguement allié sous la bannière de la lutte contre les «superstitions». L'idée générale était de faire d'une pierre deux coups : détruire les temples pour supprimer les superstitions; construire les écoles à leur place, pour promouvoir la science et le progrès. Miaochan banxue toucha l'ensemble de la société chinoise pendant plus de cinquante ans, de 1898 à l'avènement du régime communiste, même s'il est souvent considéré par les historiens seulement dans ses phases les plus actives, qui coïncident avec les flambées révolutionnaires autour de 1912 et de 1928.

Miaochan banxue se trouve à l'articulation d'un projet politique (la construction d'un système éducatif moderne) et d'un mouvement (yundong) idéologique: la lutte contre les superstitions (pochu mixin). Miaochan banxue lui-même n'est ni un véritable projet politique, ni un mouvement à proprement parler; il est trop multiforme pour cela, et utilisé par de nombreux acteurs à des fins diverses; les pratiques qui se réclament de ce terme relèvent tantôt de

1. Sous l'expression générique retenue ici de miaochan banxue (ou xingxue), j'englobe différents termes qui expriment autant de nuances. Tandis que miaochan banxue, qui semble apparaître dans des textes officiels vers 1902, désigne des procédures administratives de confiscations de biens religieux, des termes tels que huimiao banxue, ou pomiao banxue, « détruire les temple pour construire les écoles», relèvent davantage du slogan, et semblent un peu plus tardifs. 
la politique éducative, tantôt des campagnes anti-superstition. Mais, en dépit de cette diversité, il se caractérise par une nette continuité dans ses modalités et ses effets tout au long de son existence, si bien, qu'on peut le considérer comme une praxis bien identifiée. Aussi est-il question dans le présent article non des idéologies mises en œuvre dans l'appropriation des temples, mais bien du processus d'appropriation lui-même.

Les effets massifs de cette praxis, qui résulta effectivement dans la transformation de dizaines, si ce n'est de centaines de milliers de temples en écoles, sont bien connus; de fait, ils sont encore visibles aujourd'hui dans les villes et campagnes où des écoles sont installées dans des bâtiments qui de toute évidence étaient jadis les maisons de divinités. Et pourtant, si elle est souvent citée en passant par les historiens de la Chine moderne, elle n'a encore été que très peu étudiée en elle-même; elle est souvent restée comme un impensé, une évidence - pour que la Chine devienne moderne, il fallait qu'elle passe d'un pays couvert de temples à un pays couvert d'écoles; l'étude de ses modalités était dénuée d'intérêt car elle était inéluctable.

Pour tenter d'ériger miaochan banxue en véritable objet historique, et le faire passer du statut de trame de fond des récits de la modernité à celui de moteur de l'histoire, je propose ici, en m'appuyant sur les travaux détaillés existants, de l'envisager sous quatre perspectives. Cette stratégie narrative s'inspire de celle que Paul Cohen a bâtie pour faire l'histoire des Boxers (une histoire dont les rapports avec la nôtre sont d'ailleurs étroits) dans son ouvrage narré «en trois modes $»^{2}$. Mes quatre modes sont celui du processus politique, du changement socio-religieux, de l'expérience des acteurs, et de l'historiographie. Les éclairages très différents apportés par ces modes permettront d'esquisser la variété et la cohérence de miaochan banxue.

\section{Miaochan banxue comme processus politique}

L'histoire politique de miaochan banxue est partie intégrante de celle des réformes politiques et sociales de la Chine moderne depuis la fin de l'empire ${ }^{3}$. On peut trouver son origine en 1898 , lors de la réforme dite des «Cent jours » (11 juin-21 septembre). Le 10 juillet, un mémoire au trône du leader réformiste Kang Youwei (1858-1927) propose la mise en place immédiate d'un réseau d'écoles publiques sur l'ensemble du territoire en expropriant la quasi-totalité des temples (sauf ceux inscrits dans le «registre des sacrifices»,

2. Cohen, History in Three Keys.

3. Cette section reprend sous forme très condensée les analyses de Goossaert, «1898 » et Goossaert \& Palmer, Religious Question, chapitres 2 et 5. 
sidian, c'est-à-dire la liste des temples où les fonctionnaires locaux étaient tenus de sacrifier). Le mémoire est repris dans un édit impérial promulgué le jour même. Ce changement de politique était très brutal: même si auparavant l'État impérial détruisait occasionnellement des temples jugés «immoraux» $(\text { yinci })^{4}$, ces derniers ne constituaient jamais qu'une frange du paysage religieux (la destruction simultanée d'un nombre appréciable de temples dans une même région est rarissime sous les Qing), alors que la proposition de Kang Youwei remettait directement en cause l'ensemble de l'organisation de la société autour des temples de village et de quartier. Il fut jugé et salué comme l'annonce d'une révolution dans la presse et les écrits d'activistes.

Les historiens considèrent en général que le père de ce mouvement est Zhang Zhidong (1837-1909), alors gouverneur général du Hubei et Hunan, qui avait publié en mai 1898 un essai très influent et diffusé (Quanxue pian), promouvant une réforme éducative et proposant de confisquer $70 \%$ des biens des monastères bouddhiques et taoïstes à cet effet. En réalité, si les projets de Kang et de Zhang se rejoignent en partie, le second est plus conservateur dans la mesure où il visait à confisquer une partie des biens plutôt qu'à supprimer les temples, et où il visait les deux clergés plutôt que l'ensemble de la structure religieuse de la société. Quoi qu'il en soit, la réforme des Cent jours fut stoppée net et ses lois abolies dès septembre 1898, mais l'idée de s'approprier les biens des diverses communautés religieuses pour construire les écoles ainsi que (à partir de 1908) d'autres institutions de l'État-nation (postes de police, casernes, assemblées locales) continua à circuler dans la presse et dans les essais des réformateurs. La reprise de la réforme éducative dès 1901 s'accompagna d'appels à renouveler la mesure de confiscation de 1898 et de diverses initiatives locales dans ce sens; la loi de janvier 1904 sur les nouvelles écoles publiques (dont Zhang Zhidong fut l'un des auteurs) autorisait discrètement, mais sans équivoque, la saisie des biens de temples. De même la loi de 1908 sur la mise en place des bureaux d'autonomie locale (zizhi gongsuo) avait une provision similaire, qui fut également largement employée.

De fait, le mouvement de saisie commence à grande échelle vers 1902 et s'amplifie les années suivantes. Il se manifeste par des négociations tendues à l'échelle locale entre les responsables de temples et d'associations religieuses, les directeurs des nouvelles écoles, et les magistrats, débouchant souvent sur des accords prévoyant d'attribuer une partie des biens et revenus des institutions religieuses aux écoles. Outre le fait d'installer les écoles dans des bâtiments appartenant aux temples, ces derniers versaient aux écoles entre 20 et $50 \%$ de leurs revenus fonciers (ou donnaient les terres), de même que les associations

4. Goossaert, «Une répression endémique?». 
de culte (shenhui). Ces accords se présentent comme volontairement souscrits par les parties prenantes, mais ont en réalité été signés sous l'effet de contraintes et menaces. Il faut souligner que de tels arrangements étaient très différents (parce qu'imposés de l'extérieur, et marqués par une opposition idéologique entre religion et éducation) des situations, par ailleurs très courantes, où des temples, dans le cadre de leurs activités caritatives, avaient établi des écoles, des dispensaires et autres services à la communauté locale, sur leurs ressources propres et sous leur contrôle.

Là où les négociations échouent, des coups de force (saisies brutales de temples) ont lieu; ils provoquent en retour des réactions de défense voire des émeutes. Des centaines d'écoles sont attaquées et saccagées par des milices locales et des groupes religieux, mais la répartition géographique très inégale de ces épisodes violents montre que le processus de confiscation est très varié suivant les lieux. De plus, le cadre légal de ces négociations est flou; un édit impérial de 1905 recommande de protéger les biens des clergés bouddhique et taoïste, ce qui provoque la plus grande confusion et encourage les clergés à demander une réouverture des négociations, entraînant suivant les cas une baisse de leur contribution aux écoles ou un emprisonnement et une bastonnade des représentants des clergés ${ }^{5}$.

L'attitude des autorités locales est également fluctuante: tandis que certains prônent une approche conciliatrice et une vraie négociation, un bon nombre d'activistes y voient une bonne raison d'intensifier ce qu'ils considèrent comme une lutte à mort entre la superstition et le progrès. L'historiographie dominante qui s'appuie beaucoup sur les écrits des intellectuels réformateurs, qui souvent appelaient à la mesure et à la négociation, a longtemps considéré le miaochan banxue comme une simple question d'affectation de ressources ${ }^{6}$, sans mesurer les enjeux que représentent les temples, la violence du mouvement anti-superstition, et les conflits que les confiscations ont suscités dans la société locale. Un pic de violence est atteint en 1911-1912, avec l'avènement de la République, les mouvements iconoclastes qui l'accompagnent, et un brusque passage à un nouveau cadre légal pour les biens et institutions religieuses qui ne fait qu'augmenter la confusion. Dans de nombreux endroits, les fonctionnaires locaux, qui avaient jusqu'alors plutôt protégé les temples, du moins ceux avec lesquels ils entretenaient des rapports, les abandonnent voire même dans quelques cas mènent en personne des actions de vandalisme ${ }^{7}$. C'est ainsi que

5. Xu Yue, «Qingmo Sichuan miaochan xingxue».

6. Xiao-Planes, Éducation et politique.

7. Sur le terme de vandalisme pour qualifier les destructions de biens religieux orchestrées par les agents de l'État, considéré dans une perspective comparatiste, voir Goossaert \& Kouamé, «Un vandalisme d'État en Extrême-Orient?». 
des temples qui jadis se trouvaient au cœur même de la vie sociale et politique locale, comme les temples du Dieu de la Ville (chenghuangmiao), se voient attaqués en $1912^{8}$.

La multiplication des saisies, des protestations, et des procès intentés de toutes parts force le nouveau gouvernement républicain à légiférer, et à combler ainsi un vide juridique. En effet, si le code impérial affirmait le caractère intangible des biens religieux de façon générale, il ne prévoyait ni inventaire, ni règles précises pour en déterminer les droits d'usage; au demeurant, les procès entre les communautés locales possédant les temples et leurs gérants (membres des clergé bouddhiques et taoïste, notamment) étaient très fréquents sous les Qing. Une première loi, provisoire, promulguée en 1913, visait à clarifier la situation juridique des biens religieux et autorisait les pouvoirs locaux à les affecter à d'autres usages. Les temples dont les biens étaient protégés par la loi devaient relever d'une religion (concrètement, relever de l'association bouddhique ou taoïste, ou d'une des nouvelles religions reconnues par le régime); les autres, c'est-à-dire la grande majorité, ne bénéficiaient d'aucune reconnaissance, et les communautés locales (villages, quartiers) qui étaient souvent les propriétaires effectifs des temples ne furent pas reconnues comme personnes légales indépendamment de l'État.

Cette nouvelle législation provoqua une réaction des diverses associations religieuses nationales qui venaient d'être fondée pour défendre leurs intérêts ; la plus efficace et influente politiquement étant l'association bouddhique, qui constitue, par rapport aux événements d'avant 1912, un nouvel acteur du miaochan banxue. Une loi révisée, promulguée en 1915 (et amendée en 1921), précisa certains points mais continua à accorder aux pouvoir locaux le dernier mot en matière d'affectation des biens religieux ${ }^{9}$. Après 1916, les lois de la République devinrent de plus en plus lettre morte, les règlements locaux et le bon vouloir des hommes en place dictant les rapports de force, si bien qu'on assiste pendant cette période à des situations très variées, allant de saisies ponctuelles ou d'un status quo dans certains lieux à la confiscation totale des biens religieux (y compris 570 temples) et leur vente aux enchères par les autorités municipales à Guangzhou en $1923^{10}$.

La mise en place du régime nationaliste à la suite de la campagne du Nord en 1926-1928 fut l'occasion d'une nouvelle poussée de vandalisme et de législation

8. Zheng Guo, «Jindai geming yundong».

9. Siyuan guanli zhanxing guize, 20 juin 1913; Guanli simiao tiaoli, 29 octobre 1915; Xiuzheng Guanli simiao tiaoli, 20 mai 1921: voir Qu Haiyuan, «Zongjiao faling», p. 114-120.

10. Poon, Negotiating Religion, chap. 2. 
sur les biens religieux. L'activiste le plus en vue fut Tai Shuangqiu (1897-1976), un professeur en sciences de l'éducation tout juste revenu avec un doctorat de l'Université Columbia, qui fit campagne au sein des conférences nationales pour l'éducation pour des mesures radicales d'expropriation de tous les temples. Un nouvel acteur entrait dans le jeu du miaochan banxue: le Parti (Kuomintang) dont les cadres locaux furent souvent à la pointe de l'agitation contre les institutions religieuses, parfois en conflit avec les fonctionnaires plus mesurés et soucieux de maintenir l'ordre. Les lois de janvier 1929, puis de décembre 1929 sur la gestion des temples furent vivement débattues au sein même des élites dirigeantes, reflétant cette tension entre réalisme et utopie révolutionnaire ${ }^{11}$. Elles débouchèrent finalement sur une approche corporatiste offrant des garanties aux associations bouddhiques et taoïstes quant à la protection de leurs biens, mais sans jamais permettre de rouvrir les cas des biens déjà expropriés au cours des trente années écoulées. En dépit de ce cadre légal renforcé, les saisies et les procès continuèrent tout au long de la période républicaine.

\section{Le mouvement miaochan banxue comme moteur du changement socio-religieux}

L'histoire politique de la saisie des biens religieux est indispensable pour comprendre sa genèse, son évolution et ses succès. Elle tend cependant à mettre essentiellement l'accent sur les périodes de crise et sur les leaders politiques et activistes qui furent sur le devant de la scène. Considérer le mouvement comme un processus continu, bien qu'éclaté, sur la longue durée, et porter l'accent sur ses conséquences permettent de le voir sous un angle différent. Le meilleur moyen pour effectuer ce changement de perspective est sans doute de passer par une analyse quantitative. Les données sérielles pour appréhender miaochan banxue sur le terrain sont très nombreuses, mais aussi presque toujours incomplètes et difficilement comparables les unes aux autres. Les monographies locales (difangzhi) de l'époque républicaine, dans leurs sections sur les temples et sur les écoles, donnent des chiffres, plus ou moins précis, sur les biens saisis et sur la chronologie des événements. Ces données reflètent des réalités extrêmement variables; dans certains districts une large partie des temples et de leurs terres avait déjà été confisquée dès les années 1914-1915, tandis que dans d'autres les saisies ne commencent à grande échelle que quinze ans plus tard. Les types de temples visés ne sont pas toujours les mêmes non plus. Et, la part des biens religieux dans l'effort global de construction des écoles varie, même si l'on

11. L'analyse la plus complète de la gestion des temples sous le régime de Nankin est Nedostup, Superstitious Regimes, chap. 3. 
trouve généralement plus de la moitié des écoles soit utilisant des locaux d'un temple soit ayant récupéré les biens fonciers d'une institution religieuse ${ }^{12}$.

Pour se faire une première idée du phénomène à une échelle locale, je me suis appuyé sur des listes très riches (même si très certainement incomplètes) de 639 temples dans l'actuelle municipalité de Shanghai (comprenant donc aussi les districts ruraux entourant la ville proprement dite), fournissant des informations sur leur destin au $\mathrm{xx}^{\mathrm{e}}$ siècle ${ }^{13}$. Ces listes fournissent la matière d'une analyse chronologique des appropriations, et d'une différenciation entre divers types de temples. Les graphiques ci-dessous représentent une synthèse de ces données; chaque temple y figure l'année de la première appropriation connue (même si dans certains cas, le temple a continué à fonctionner sur une échelle réduite et a connu des appropriations ultérieures) ${ }^{14}$.

\section{Graphique 1. Destruction des temples bouddhiques de Shanghai}

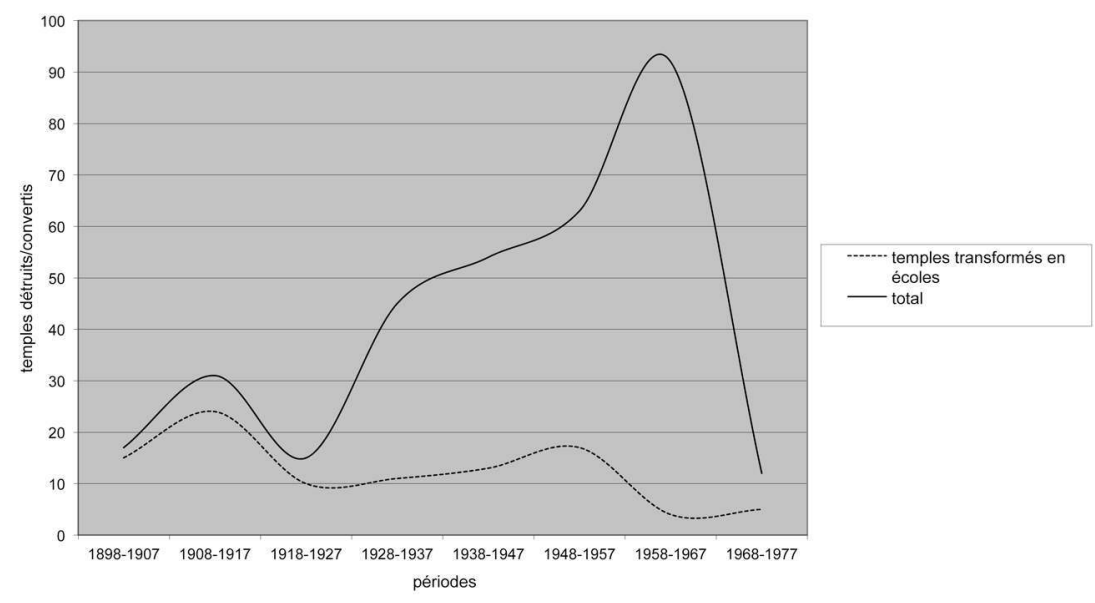

12. Liang Yong, «Qingmo "miaochan xingxue”», p. 112, montre que déjà en 1908, 56 \% des écoles de Baxian recevaient des fonds saisis aux institutions religieuses, et $75 \%$ étaient installées dans des temples; Xiong Chunwen, «Wenzi shangyi», p. 13, cite des chiffres comparables pour le Zhejiang et d'autres régions. Voir aussi Goossaert \& Palmer, Religious Question, chap. 5.

13. Zhang Hua, Shanghai zongjiao tonglan, p. 22-182 (temples «bouddhiques», au sens le plus large) et p. 228-329 (temples «taoïstes», au sens le plus large): ces listes comprennent 639 temples pour lesquels la date de l'appropriation est bien documentée, parmi de nombreux autres non retenus ici.

14. Je n'ai pas tenu compte des écoles établies par les bouddhistes eux-mêmes, et j'ai lissé les données qui donnent parfois des dates approximatives en les regroupant par périodes de dix ans. 


\section{Graphique 2. Destruction des temples taö̈stes de Shanghai}

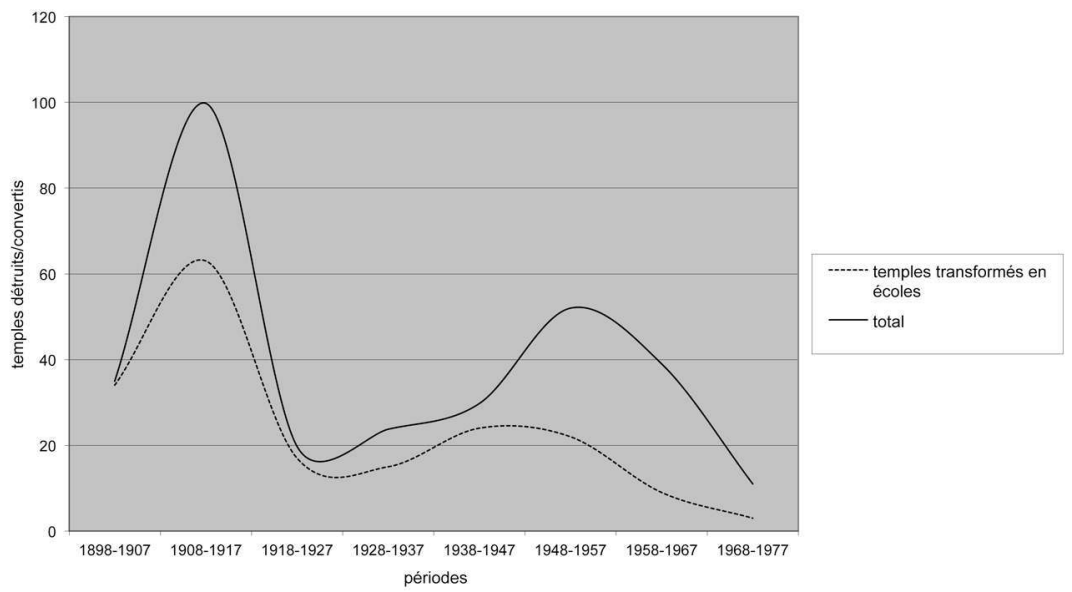

Graphique 3. Conversion de temples en écoles, Shanghai

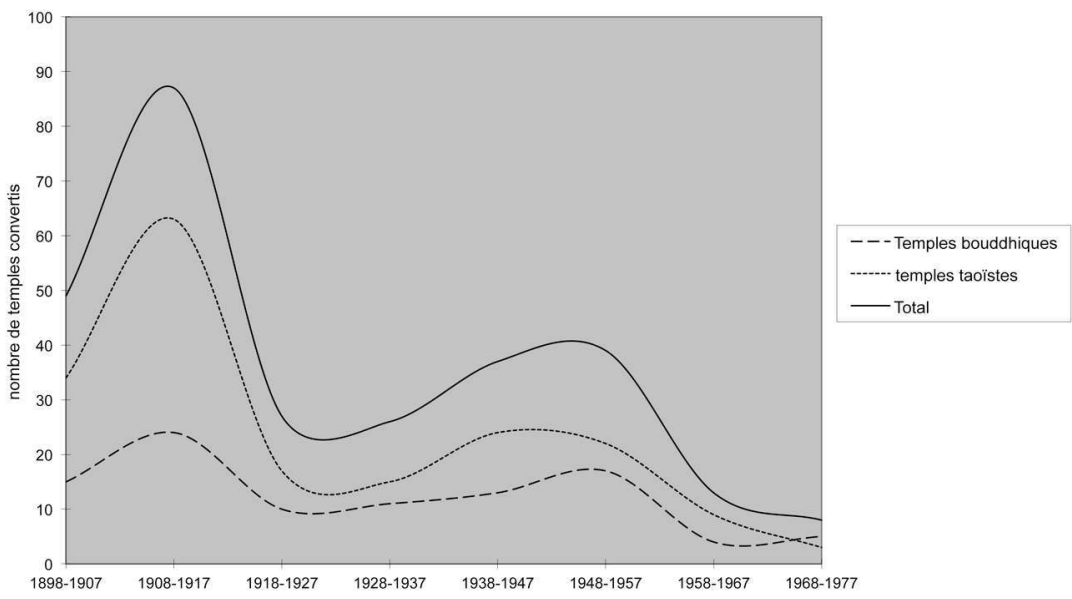

Il ressort de ces graphiques que la saisie des temples (les données utilisées ne reflètent pas la saisie des biens des associations) dans cet ensemble géographique complexe ne s'est jamais arrêtée pendant plus de cinquante ans, et se prolonge sans solution de continuité pendant le début de la période communiste. Si les pics identifiés par l'histoire politique $(1912,1928)$ se reflètent nettement par une augmentation dans les appropriations à Shanghai (ce qui n'est pas nécessairement le cas pour d'autres lieux), on constate néanmoins que des temples furent appropriés chaque année, et que ces appropriations relèvent donc autant d'une 
évolution de la société locale que d'événements politiques nationaux. Bien que les acteurs, le contexte légal et idéologique aient été différents en 1905, dans les années 1910 et après 1927, cette continuité permet d'envisager le miaochan banxue comme un processus cohérent. En revanche, si la transformation en écoles demeure la cause dominante d'appropriation des temples jusqu' au début des années 1930, d'autres types de transformation (casernes, usines, bureaux, et de plus en plus, destruction pure et simple) deviennent majoritaires par la suite.

Par ailleurs, on constate que les temples classés par notre source comme «taoïstes» (essentiellement des temples communautaires des villages et quartiers) ont été saisis plus tôt et davantage que ceux classés comme bouddhiques (pour l'essentiel possédés et gérés par le clergé), certainement parce que les seconds offraient une résistance plus efficace et mieux organisée, notamment via l'association bouddhique à partir de 1912. Les saisies des monastères ont été davantage documentées et débattues que celles des temples de quartier, de lignage, de guildes, et des associations de culte, mais elles n'en sont pas pour autant représentatives de l'ensemble du phénomène miaochan banxue.

Ainsi, plutôt qu'une série de crises, la lecture de ce graphique invite à voir dans le mouvement miaochan banxue un glissement progressif s'étalant sur deux générations, d'un modèle de société organisé autour de temples de village, dotés de biens fonciers, vers un autre modèle, avec d'une part un État beaucoup plus présent, mais aussi d'autres types d'institutions religieuses indépendantes des structures de pouvoir locales. Car, si les analyses existantes du miaochan banxue évoquent la destruction des institutions et de leurs ressources, elles évoquent aussi les opportunités ouvertes pour de nouvelles formes d'organisation religieuse: bon nombre de travaux évoquent le réformisme bouddhique qui a prospéré dans un contexte de remise en question de l'économie monastique traditionnelle ${ }^{15}$. Mais il faut aussi évoquer les sociétés rédemptrices, ces nouveaux groupes religieux caractéristiques de l'époque républicaine qui se multiplient alors ${ }^{16}$ en fondant leurs propres temples, souvent en récupérant d'anciens temples ruinés par la saisie de leurs terres et en adoptant un autre modèle économique (incorporation légale, peu de biens fonciers, revenus essentiellement dérivés des cotisations mensuelles des membres) plus adapté au contexte politique et légal - les sociétés rédemptrices telles que le Daode xueshe ou le Tongshanshe construisirent aussi un très grand nombre d'écoles.

15. Sur le réformisme bouddhique, voir Pitman, Toward a Modern Chinese Buddhism; pour une discussion du rapport direct entre la destruction des temples et l'essor du réformisme, voir Liu Chengyou \& Mei Haizi, «Miaochan xingxue yu fojiao gexin».

16. Sur les sociétés rédemptrices, voir le double numéro 172-173 à paraître de la revue Min-su chü-i, et Goossaert \& Palmer, Religious Question, chap. 4. 
De l'époque Qing à la période républicaine, le modèle économique dominant (auquel se conforme la plupart des temples) connait une mutation de grande ampleur: la propriété collective au nom des dieux ou des ancêtres et investissant dans la terre fait place à l'entreprise privée investissant dans les biens financiers ${ }^{17}$. Si les biens des associations de culte sont saisis, alors les guildes se transforment en chambres de commerce et en associations professionnelles, et les tudihui en associations de quartiers. Un résumé saisissant de cette mutation est fourni par Hong Kong qui adopte en 1928 une loi nationalisant tous les temples traditionnels de quartier (dont les revenus sont reversés aux hôpitaux et autres œuvres caritatives), loi exigée des autorités coloniales par les élites marchandes désireuses d'imiter les politiques du Parti nationaliste ${ }^{18}$. À partir de cette date, les temples traditionnels déclinent fortement, et toute la vitalité religieuse du territoire se porte vers des temples privés fondés par des groupes enregistrés comme associations caritatives ou comme entreprises commerciales.

\section{Miaochan banxue comme expérience des acteurs historiques}

L'approche politique comme l'approche d'histoire socio-religieuse voient le miaochan banxue comme un macro-phénomène. Qu'en est-il de l'expérience des acteurs qui y participèrent, comme activistes, leaders, enseignants ou comme simple écoliers? Vu sur ce mode, miaochan banxue n'apparaît plus comme une vague emportant la société chinoise dans son ensemble, mais comme un agrégat de situations uniques.

De ce point de vue, l'une des premières questions qui se posent est celle des contraintes et du niveau de violence du processus d'appropriation. Toute la gamme de situations a existé, depuis la saisie brusque et violente avec vandalisme et violence sur les personnes (comme la saisie du temple Tieshansi à Pékin en 1931 par des ouvriers endoctrinés par une cellule du Parti nationaliste ${ }^{19}$ ), jusqu'à un partage volontaire où les responsables d'un village décident d'eux-mêmes de répartir les ressources collectives entre temple et école en tentant autant que possible de préserver les intérêts des deux. Mais ces deux extrêmes ne représentent ni l'un ni l'autre la majorité des cas, qui semblent plutôt relever de négociations, généralement tendues et faisant entrer en ligne de compte divers rapports de force (menaces de la part des autorités, chantages), mais évitant le recours à la violence physique (destruction des statues, textes, et autres biens religieux, contraintes sur les personnes). Le niveau de violence dépendait en

17. C'est la thèse de Faure, Emperor and Ancestor, chap. 22.

18. Goossaert \& Palmer, Religious Question, chap. 7.

19. Pour une étude détaillée de ce cas, voir Fu Haiyan, «Geming, falü yu miaochan». 
partie du contexte politique global, mais négociations et rapports de forces étaient eux propres à chaque contexte local.

Vues de près, ces négociations font souvent figurer les institutions religieuses comme des acteurs qui, loin de subir les événements comme le veut l'historiographie dominante, y jouent un rôle décisif. D'une part, des temples construisirent leurs propres écoles modernes - des temples bouddhiques ${ }^{20}$, mais aussi taoïstes, comme dans le cas de Nanyang (sud du Henan) où comme le montre Liu Xun dans ce volume, la majorité des élèves jusque dans les années 1920 fréquentaient des écoles financées et gérées par le grand monastère taoïste de la ville. Par ailleurs, dans la majorité des cas où la gestion des écoles était entre les mains d'éducateurs laïques, un accord était signé avec les institutions religieuses. Ainsi, à Baxian (actuel Chongqing), un accord fut signé fin 1904 par tous les temples du district, qui versaient tous une contribution financière (miaojuan) correspondant à $20 \%$ de leur revenu à un bureau géré par le responsable officiel du clergé bouddhiste, qui le reversait aux écoles ${ }^{21}$. Mais, tous ces temples et congrégations étaient déjà appauvris et les sommes issues de ces cotisations étaient modestes, très loin des fantasmes des activistes qui imaginaient les fortunes des institutions religieuses, si bien que les fonctionnaires n'eurent de cesse d'augmenter leur montant, ruinant ainsi par le haut les efforts de conciliation trouvés dans la société locale. Autant que le début des appropriations, le moment de rupture a été celui du passage (ou de la prise de conscience du passage), à des moments différents en chaque lieu, en dessous du niveau de revenu des biens collectifs où la vie religieuse devenait dramatiquement réduite, l'entretien des temples inabordable, et les célébrations collectives impossibles; ce passage a provoqué chez beaucoup d'acteurs des basculements, pour ou contre les écoles, ou les temples. Par ailleurs, l'effet du miaochan banxue sur la vie rituelle et festive, et sur l'intégration sociale des communautés locales reste entièrement à étudier. Qu'en était-il de l'attitude des directeurs et maîtres d'école dans la vie du village? Certains étaient des activistes de l'anti-superstition, mais d'autres (ou les mêmes) étaient membres de groupes religieux (entre autres des sociétés rédemptrices). Quand il tente de mettre fin aux grandes processions des temples dans la région de Shanghai après 1927, le Parti nationaliste envoie les maîtres d'école en première ligne à côté des policiers, pour convaincre les leaders d'annuler les fêtes, ce qui (outre les bagarres et les incidents graves) leur vaut d'avoir des écoles vandalisées

20. He Jinlin, «Qingmo seng jiaoyuhui».

21. Liang Yong, «Qingmo «miaochan xingxue»», p. 111. Cette procédure était répandue dans le Sichuan avant 1911: Xu Yue, «Qingmo Sichuan miaochan xingxue». 
en retour ${ }^{22}$. Il n'est pas sûr que tous les enseignants aient apprécié d'être ainsi utilisés par le régime et qu'ils aient souscrit à cette vision qui leur était imposée par le Parti, de l'école contre les temples.

Et, au-delà de l'expérience qu'a constitué, pour les chefs de village, responsables de temples et autres acteurs locaux, le fait d'avoir à gérer cette politique imposée par le haut de construire des écoles dans l'urgence et par tous les moyens, reste à comprendre l'expérience plus commune encore qu'a constitué le fait d'aller à l'école, d'apprendre entre autres choses l'athéisme scientifique, dans un lieu où l'on venait jadis brûler de l'encens, et où souvent les femmes âgées continuaient à venir brûler de l'encens, devant la porte ou dans la cour, si ce n'est dans la salle de classe.

\section{L'historiographie du miaochan banxue}

Alors que dans l'histoire de l'Europe la relation tendue entre l'école et la paroisse (ou entre le prêtre et l'instituteur) a été très largement étudiée ${ }^{23}$, miaochan banxue est très longtemps resté un impensé de l'historiographie de la Chine moderne. La glorification des réformistes et révolutionnaires rendait difficile l'étude des violences et destructions ayant accompagné la construction de l'État-nation. Les historiens du système éducatif ont eu tendance à penser que la transformation des temples en écoles s'était faite naturellement, volontairement, ce qui est loin d'être vrai dans la majorité des cas. Pendant longtemps, les seuls historiens à avoir problématisé la saisie des temples furent les historiens du bouddhisme, qui en firent le contexte de l'émergence du réformisme moderne ${ }^{24}$. Si ces historiens ont les premiers documenté le mouvement miaochan banxue, ils l'ont aussi interprété dans un sens particulier (spécifiquement anti-bouddhique, ce qui n'est vrai que d'une partie des actions de saisie), et ils lui ont donné une dimension téléologique (permettre au bouddhisme réformé de s'affirmer). Mais, depuis les années 1990, l'influence considérable (en Chine autant qu'en Occident) des travaux de Prasenjit Duara, sur l'intrusion de l'État dans la société villageoise et la destruction du cultural nexus of power (où les temples occupent un rôle central) qui en a résulté ${ }^{25}$, a permis de jeter les bases théoriques de nouveaux travaux en Chine même ${ }^{26}$.

22. Ai Ping, «Minguo jinzhi yingshen saihui lunxi»,p. 218.

23. Par exemple, Ozouf, L'École, l'Église et la République.

24. Makita, «Seimotsu irai»; Shi Dongchu, Zhongguo fojiao jindai shi, p. 72-77. Pour les besoins du présent article ne sont cités ici qu'un tout petit nombre parmi les nombreux travaux récents portant sur le miaochan banxue.

25. Duara, Culture, Power, and the State; Rescuing History from the Nation.

26. Par exemple, Shen Jie, «Xiandaihua jianzhi». 
Depuis le début des années 2000, une nouvelle génération d'historiens a commencé à prendre au sérieux les conflits et tensions provoqués par la saisie des temples, y voyant un élément important des changements sociaux induits par la construction de l'État-Nation. Là où pour les historiens antérieurs les mouvements de résistance étaient forcément marginaux et rétrogrades, on y voit désormais des élites très divisées sur la compréhension de leurs propres intérêts. Cet intérêt nouveau est peut-être lié au fait que désormais certains temples récupèrent leurs locaux longtemps occupés par les écoles (quand celles-ci sont agrandies et déplacées vers de nouveaux sites, ou juste déplacées pour mettre en valeur un site touristique), ce qui rend le cours de l'histoire moins univoque, et la question plus ouverte.

Dans son article sur le miaochan banxue dans la région de Chongqing avant 1911, appuyé sur les archives de Baxian, Liang Yong montre que le mouvement s'est appuyé sur une partie des élites locales qui se trouvaient marginalisées dans le contrôle des institutions locales (temples, fondations charitables, guildes) qui levaient des taxes et contrôlaient les marchés ${ }^{27}$. Ces personnes s'engouffrèrent dans le statut nouveau de directeur d'école ou de responsable de l'éducation pour un canton (nommé par le magistrat et non désigné localement); les magistrats, les règlements impériaux et locaux les encouragèrent à s'approprier les ressources des institutions locales, attisant ainsi les tensions. Les magistrats eux-mêmes ne se mêlèrent pas directement de la gestion des écoles (sauf pour les plus grandes). Liang Yong remarque aussi que les fonds des écoles étaient beaucoup moins contrôlés que ceux des temples, créant ainsi de nombreuses opportunités de corruption. Pour autant, les accusations de corruption, de dissimulation des revenus et de partialité volaient dans les deux sens, et servaient d'armes dans les bras de fer et négociations pour le partage des ressources locales ${ }^{28}$. Les négociations qui aboutissaient donnaient lieu à un partage relativement équilibré, mais en cas d'échec, elles arrivaient devant le magistrat qui donnait systématiquement l'avantage aux écoles. La coloration très juridique de ces sources d'archives, où l'idéologie est souvent reléguée en toile de fond, fait qu'une partie des travaux récents se placent dans la perspective de l'histoire du droit de la propriété, remontant aux conflits de l'époque impériale sur les biens religieux, et tentant de faire l'histoire des conceptions et pratiques des biens collectifs (gong) dont les temples sont une partie très importante ${ }^{29}$.

27. Liang Yong, «Qingmo «miaochan xingxue»».

28. Liang Yong, ibid., p. 114 donne l'exemple d'un gérant d'école accusant un gérant de temple de corruption puis signant avec lui un accord partageant en deux les revenus des terres du temple.

29. Fu Haiyan, «Geming, falü yu miaochan», p. 106, n.1. 
Par ailleurs, la nouvelle génération d'historiens, plutôt que d'envisager la saisie des temples globalement comme un phénomène idéologique, examine des cas spécifiques et des procès, à partir de fonds d'archives ou de la presse; la plupart de ces cas concernent les périodes 1902-1908 et 1928-1932, laissant l'époque Beiyang, 1916-1928, très sous-étudiée. Ces études de cas permettent de dépasser une vision simple d'une opposition entre des élites modernisatrices et une société locale conservatrice, et de mettre en évidence les rôles complexes joués par toutes sortes d'acteurs dont on entend ainsi les voix, jadis couvertes par les discours idéologiques. Ces exemples montrent que le contexte politique et les idéologies mises en œuvre (progrès, anti-superstition, liberté religieuse) servent souvent de prétexte ou de cartes à jouer dans des négociations complexes. Dans les textes des acteurs se mêlent toutes sortes d'arguments : les éducateurs parlent dès 1902 de convertir l'inutile (wuyi) en utile (youyi) (les temples en écoles), un vocabulaire déjà courant dans les années 1870 pour critiquer les grandes fêtes populaires, et qui intègre le discours dans la loi sur les écoles de janvier 1904; les responsables de temples invoquent le code impérial ou les lois républicaines en faveur de la liberté religieuse. Tous les acteurs, à chacune des étapes du miaochan banxue, ont su manipuler le vocabulaire du moment à leurs propres fins.

De ce fait, les travaux les plus récents remettent en question les convictions des acteurs historiques quand aux cultes, aux rites et à l'enseignement, déterminant leurs choix quant aux temples et aux écoles, convictions («conservatrices» ou «modernistes ») qui ont longtemps semblé évidentes aux historiens. Cette question renvoie à la religiosité des élites locales, à leur culture religieuse et leur participation à la vie rituelle locale, qui furent très variées et expliquent dans une mesure importante la diversité des formes locales du miaochan banxue ${ }^{30}$. Et l'identification des leaders des temples à des «membres de la gentry» ne suffit plus; il faut des catégories plus fines, et une idée plus précise de la part des élites locales prenant encore activement part à la gestion des temples locaux en 1898 ou en 1911.

\section{Conclusion}

L'histoire du miaochan banxue reste encore à écrire; les grandes lignes du processus politique et du contexte idéologique sont connues, mais les contours et les effets du phénomène, dans sa durée et dans sa variété géographique, restent très flous. Une multiplication très récente de travaux historiques portant sur des cas précis vient jeter les bases d'une telle histoire, montrant la complexité

30. Pour un cas d'espèce, voir Goossaert, «Yu Yue (1821-1906) explore l'au-delà». 
du phénomène. Si le mode de l'histoire politique a été favorisé, les modes de l'expérience et du changement socio-religieux permettront de l'appréhender comme un objet historique à part entière.

Somme toute, l'un des intérêts principaux de cet objet est de reposer la question, au cœur du présent volume, des rapports entre religion et éducation. Car, si chaque cas particulier de saisie des biens religieux met en jeu des facteurs spécifiques (luttes entre diverses sections des élites locales, ou entre le parti et le gouvernement local, contrôle des biens communautaires, etc.), la question qui demeure en arrière-fond est que l'école et le temple occupent le même espace au sein de la société locale, celui du contrôle des ressources symboliques et matérielles de la communauté et donc de la source de toute légitimité pour l'exercice du pouvoir. Or, si le discours idéologique a souvent argumenté que l'un et l'autre sont exclusifs dans cet espace, la réalité du processus historique est aussi en partie celle d'une âpre négociation et d'un partage. On trouve des membres des élites locales qui tout en promouvant les écoles défendent les temples locaux ${ }^{31}$. Le fait que les maîtres et directeurs d'école aient contribué à reconstruire les temples après 1978 en dit long sur le partage de cet espace commun; de fait, depuis 1898 , les débats et procès successifs montrent que les deux côtés - écoles et temples - partagent un même vocabulaire, celui du service public, du bien commun, du dévouement, et que le temple et l'école sont deux lieux où ce vocabulaire et son imaginaire se déploient.

\section{Bibliographie}

AI Ping 艾萍, «Minguo jinzhi yingshen saihui lunxi. Yi Shanghai wei gean»民國 禁止迎神賽會論析一一上海為個案, Jiangsu shehui kexue 江蘇社會科學 5, 2010, p. 216-221.

CoHen Paul A., History in Three Keys: The Boxers as Event, Experience, and Myth, New York, Columbia University Press, 1997.

Dean Kenneth \& Zheng Zhenman, Ritual Alliances of the Putian Plain, Leyde, Brill, 2010.

Duara Prasenjit, Culture, Power, and the State: Rural North China, 1900-1942, Stanford, Stanford University Press, 1988.

- -, Rescuing History from the Nation: Questioning Narratives of Modern China, Chicago, University of Chicago Press, 1995.

Fu Haiyan 付海晏, «Geming, falü yu miaochan - Minguo Beiping Tieshansi an yanjiu» 革命、法律與廟產——民國北平鐵山寺案研究, Lishi yanjiu 歷史研 究 3, 2009, p. 105-120.

31. Dean \& Zheng, Ritual Alliances, vol. 1, p. 139-143. 


\section{Vincent Goossaert}

GOOSSAERT Vincent, «1898: The Beginning of the End for Chinese Religion?», Journal of Asian Studies 65 (2), 2006, p. 307-336.

- - , «Une répression endémique? La destruction des "temples immoraux" en Chine sous les Qing», in Nathalie KouAmé, Arnaud Brotons, Yannick Bruneton (dir.), État, religion et répression en Asie. Chine, Corée, Japon, Vietnam (XIII ${ }^{e}-$ XXI $I^{e}$ siècles), Paris, Karthala, 2011, p. 183-222.

— - «Yu Yue (1821-1906) explore l'au-delà. La culture religieuse des élites chinoises à la veille des révolutions », in Roberte HAMAYON, Denise AIGLE, Isabelle CHARLEUX \& Vincent Goossaert (dir.), Miscellanea Asiatica, Sankt Augustin, Monumenta Serica, 2011, p. 623-656.

GoOSSAERT Vincent \& Nathalie KOUAMÉ, «Un vandalisme d'État en Extrême-Orient? Les destructions de lieux de culte dans l'histoire de la Chine et du Japon », Numen 53 (2), 2006, p. 177-220.

Goossaert Vincent \& David A. Palmer, The Religious Question in Modern China, Chicago, University of Chicago Press, 2011.

HE Jinlin 賀金林, «Qingmo seng jiaoyuhui yu siyuan xingxue de xingqi » 清末僧教育 會與寺院興學的興起, Anhui shixue 安徽史學 6,2005,p. 28-34.

LIANG Yong 梁勇, "Qingmo “miaochan xingxue” yu xiangcun quanshi de zhuanyi - yi Baxian wei zhongxin» 清末 “廟產興學” 與鄉村權勢的轉移一一以巴縣為 中心, Shehuixue yanjiu 社會學研究 1, 2008, p. 102-119.

Liu Chengyou 劉成有 \& MEI Haizi 梅海子, «Miaochan xingxue yu fojiao gexin» 廟 產興學與佛教革新, Xuzhou shifan daxue xuebao (Zhexue shehui kexue ban) 徐 州師範大學學報(哲學社會科學版) 30 (3), 2004, p. 93-96.

MAKITA Tairyô 牧田諦亮, «Seimotsu irai ni okeru byôsan kôgaku» 清末以來に於 ける廟產興學, In Chûgoku bukkyôshi kenkŷ̂ 中國佛教史研究, vol. 2, Tokyo,

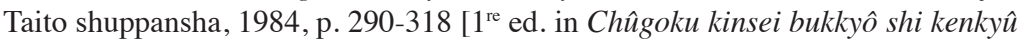
中國近世佛教史研究, Kyoto, Heirakuji shoten, 1957].

Nedostup Rebecca A., Superstitious Regimes: Religion and the Politics of Chinese Modernity, Cambridge, Harvard University Asia Center, 2009.

Ozouf Mona, L'École, l'Église et la République (1871-1914), Paris, Seuil, 2007.

Pitman Don A., Toward a Modern Chinese Buddhism. Taixu's Reforms, Honolulu, University of Hawai'i Press, 2001.

Poon Shuk Wah, Negotiating Religion in Modern China: State and Common People in Guangzhou, 1900-1937, Hong Kong, Chinese University Press, 2011.

Qu Haiyuan 翟海源, «Zhonghua minguo youguan zongjiao faling ji falü caoan huibian»中華民國有關宗教法令及法律草案彙編, Minzuxue yanjiusuo ziliao huibian 民族學研究所資料彙編, 2, 1990, p. 113-139.

SHEN Jie 沈潔, «Xiandaihua jianzhi dui xinyang kongjian de zhengyong - yi ershi shiji chunian de miaochan xingxue yundong weili» 現代化建制對信仰空間的 征用一一以二十世紀初年的廟產興學運動為例, Lishi jiaoxue wenti 歷史教學 問題 2, 2008, p. 56-59.

SHI Dongchu 釋東初, Zhongguo fojiao jindai shi 中國佛教近代史, Taibei, Dongchu chubanshe, 1974.

Xiao-Planes Xiaohong, Éducation et politique, Paris, EHESS, 2001. 
XIONG Chunwen 熊春文, «"Wenzi shengyi”: 20 shiji 90 niandaimo yilai Zhongguo xiangcun jiaoyu de xin quxiang»“文字上移” : 20世紀90年代末以來中國鄉 村教育的新趨向, Shehuixue yanjiu 社會學研究 5, 2009, p. 1-32.

XU Yue 徐躍, «Qingmo Sichuan miaochan xingxue ji youci chansheng de sengsu jiufen» 清末四川廟產興學及由此產生的僧俗糾紛, Jindaishi yanjiu 近代史研 究 5, 2008, p. 73-88.

ZhANG Hua 張化, Shanghai zongjiao tonglan 上海宗教通覽, Shanghai, Shanghai guji chubanshe, 2004.

ZHENG Guo 鄭國, «Jindai geming yundong yu pochu mixin - yi xuzhou chenghuangmiao weizhu de kaocha» 近代革命運動與破除迷信——以徐州城隍廟為主的考察, Hefei shifan xueyuan xuebao 合肥師範學院學報 26 (2), 2008, p. 54-57.

\section{GLOSSAIRE}

chenghuangmiao 城隍廟

Daode xueshe 道德學社

gong 公

huimiao banxue 毀廟辦學

Kang Youwei 康有為

Kuomintang 國民黨

miaochan banxue (xingxue) 廟產辦 (興) 學

miaojuan 廟捐

pochu mixin 破除迷信

pomiao banxue 破廟辦學

shenhui 神會

sidian 祀典

Siyuan guanli zhanxing guize 寺院管理暫行規則

(Xiuzheng 修正) Guanli simiao tiaoli 管理寺廟條例

Tai Shuangqiu 邰爽秋

Tongshanshe 同善社

tudihui 土地會

wuyi 無益

yinci 淫祠

youyi 有益

yundong 運動

zizhi gongsuo 自治公所

Zhang Zhidong 張之洞 
